\title{
Eddy Current Modelling Using the Impedance Method for Surface Impedance Profiling
}

Daniel A. James Student Member, IEEE, Steven G. O'Keefe, Member, IEEE, and David V. Thiel, Senior Member, IEEE

\begin{abstract}
In this paper a new technique for determining surface impedance is developed. The technique is derived from an eddy current modelling technique, the impedance method. Considerable extension and development of the impedance method has been undertaken, enabling the prediction of surface impedance measurements for horizontally stratified media and in the vicinity of vertical dislocations. The method is compared with surface impedance theory for horizontally stratified media and with field based results near laterally discontinuous structures.
\end{abstract}

Index terms-eddy currents, surface impedance, impedance method

\section{INTRODUCTION}

Surface impedance measurements are used in geophysical surveying for the determination of sub-surface features. Surface impedance theory allows the determination of subsurface structure from these measurements and has been used to deduce material thickness and conductivity in ice covered regions [1][2] and in the mineral exploration industry for lateral anomaly location (eg. faults, dykes, ore boundaries) [3]. The existing theory does not adequately explain the rapid changes caused by complex earth structures.

An examination of surface impedance data has revealed that the primary component of the excursions near anomalies was primarily from the electric field component produced by telluric eddy currents. These currents are cited as being induced by the sub-surface magnetic field [4] and hence eddy current modelling may predict the anomalous behaviour.

Three dimensional impedance methods have been used to model eddy currents induced in heterogeneous human models by a time varying quasi-static magnetic field [5][6]. These methods use regular mesh elements to model well bounded regions for eddy currents. The methods have been extended previously by the author to model partially unbounded structures using irregular mesh elements and spatially variable fields [7]. In this paper, these concepts are extended to surface impedance calculations, with numerical results compared with experimental measurements.

Manuscript received June 3, 199; revised January 25, 1999

Corresponding author David V. Thiel. $+(617)-3875-7192$, fax $+(617)$ 3875 -5198. dthiel@me.gu.edu.au,

The authors are with the Radio Science Laboratory, School of Microelectronic Engineering, Griffith University, Nathan Qld 4111 Australia.

\section{SURFACE IMPEDANCE THEORY}

A vertically polarised radio wave incident upon the surface of the earth induces a horizontal electric field component $E_{X}$ directed in the plane of incidence (Fig. 1). For a uniform half space, the ratio of this field component to the magnetic field component $H_{y}$ perpendicular to the plane of incidence is called the surface impedance $Z_{S}$. The surface impedance is dependent on the electrical properties of the half space and is given by (1).

$$
\mathrm{Z}_{\mathrm{S}}=\frac{E_{x}}{H_{y}}=\sqrt{\frac{j \omega \mu}{\sigma+\varepsilon \omega}}
$$

where $\sigma$ is the conductivity, $\varepsilon$ is the relative permittivity and $\omega$ is the angular frequency. If the earth is horizontally layered, then $Z_{S}$ for the upper layer (assuming an infinite half space) is modified as [8].

$$
\begin{aligned}
& Z_{S}=Q_{1} Z_{1} \\
& Q_{1}=\frac{Z_{2}+Z_{1} \tanh \left(u_{1} h\right)}{Z_{1}+Z_{2} \tanh \left(u_{1} h\right)} \\
& u_{1}=\sqrt{\gamma_{1}^{2}-\gamma_{1}^{2} \sin ^{2} \phi}
\end{aligned}
$$

where $u_{I}$ is the complex propagation co-efficient $\gamma_{0}$ is the free space propagation co-efficient $\gamma_{1}$ is the propagation co-efficient in the first layer $\phi$ is the angle of incidence, and $h$ is the depth of the upper layer

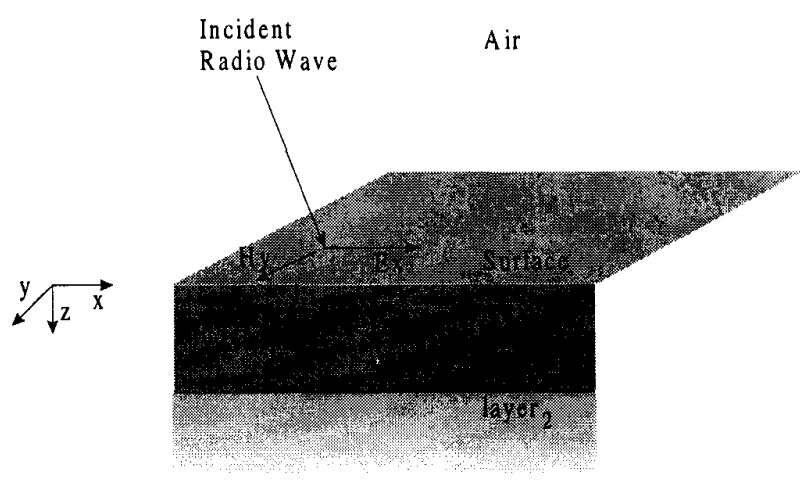


Fig. 1. Incident radiation on a two layer half space
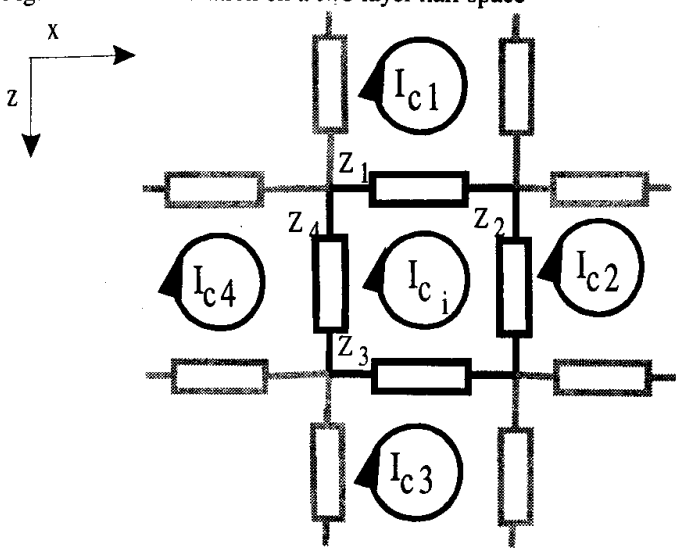

III. IMPEDANCE METHOD THEORY

This section reviews the basic impedance method theory, boundary conditions, using propagating waves as the source and the derivation of surface impedance from the eddy current solutions.

\section{A. Basic Theory}

The region of interest to be modelled is discretised into a mesh of impedance elements. Each enclosed area forms a cell through which an applied flux induces cell current $I_{\mathbf{C}_{\mathbf{i}}}$ (Fig. 2).

The impedance of each element $Z$ is determined by its length $\mathrm{L}$, area $\mathrm{A}$, and material properties $(\sigma, \varepsilon)$.

$$
\mathrm{Z}=\frac{L}{\left(\sigma+j \omega \varepsilon_{r} \varepsilon_{0}\right) \mathrm{A}}
$$

The applied flux $\Phi_{i}$ induces a potential $V_{Z_{j}}$ in each cell loop

$$
\mathrm{j} \omega \Phi_{\mathrm{i}}=\sum_{j=1}^{4} V_{z_{j}}
$$

where $Z_{i}$ is the impedance of the $j^{\text {th }}$ element.

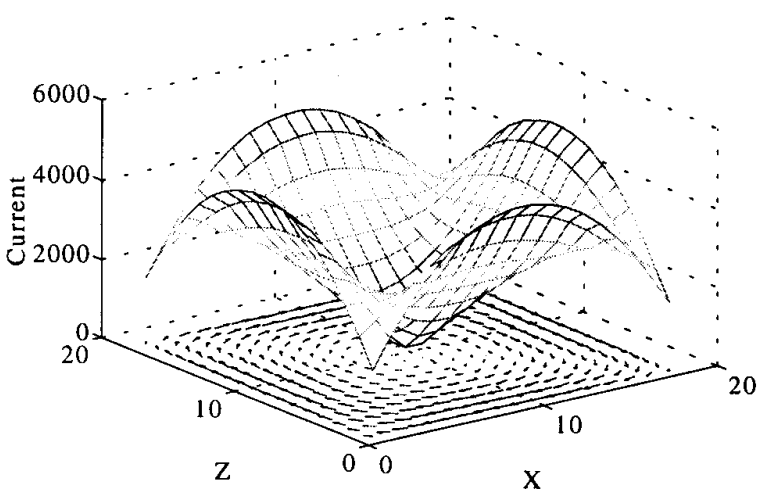

Fig. 3. Induced currents in a square conducting sheet from a uniform quasistatic magnetic field. Using Ohm's law the cell currents are calculated

$$
\mathrm{j} \omega \Phi_{\mathrm{i}}=\sum_{j=1}^{4} I_{C_{i}} Z_{j}
$$

Hence the relationship between one cell $I_{c_{i}}$ and its nearest neighbour cell currents $I_{c j}$ is given by

$$
j \omega \Phi_{c_{i}}=\sum_{j=1}^{4}\left(I_{C_{i}}-I_{C_{j}}\right) Z_{j}
$$

For a conductive sheet consisting of $\mathrm{n}$ cells, the mesh can be represented by

$$
[\mathrm{Z}]_{\mathrm{nxn}} \cdot[\mathrm{I}]_{1 \mathrm{xn}}=\mathrm{j} \omega[\Phi]_{1 \times \mathrm{xn}}
$$

where $[Z]$ forms the impedance network, $[\mathrm{I}]$ is the column matrix of unknown currents to be calculated and $[\Phi]$ is a row matrix of magnetic flux through each element.

A conducting copper sheet is modelled with $18 \times 18$ cells and produces a well known circulating current about the centre point (Fig. 3).

\section{B. Boundary Extensions}

The method has been extended to enable the modelling of small regions of interest within boundaries extending to infinity and to encompass spatially variable magnetic field source problems by application of the Neumann boundary condition [7]. This allows the modelling of infinite half space problems where the field source is attenuated by the media. A uniform material has the magnetic field exponentially attenuated as $\mathrm{Z}$ increases (Fig. 4) in an $18 \times 18$ cell model.

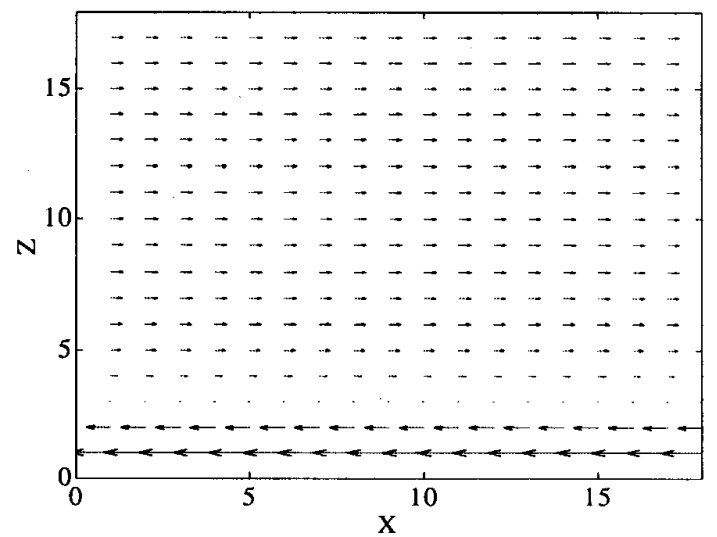

Fig. 4. Induced currents in an infinitely long conductive strip from an applied exponentially spatially variant magnetic field $\mathrm{H}_{\mathrm{y}}(\mathrm{z})$. 


\section{Wave Propagation Extensions}

An extension upon this method enables the calculation of a complex magnetic field as it propagates through a sub-surface using the usual equations for plane wave dielectric materials (Fig. 5(a)). For a multilayer sub-surface reflected and transmitted components need to be calculated (Fig. 5(b)).

By solving a one dimesional model for a single layer subsurface, the induced currents can be examined (see Fig. 6) as a function of depth. Clearly as the model depth increases the currents decrease. For models that exceed one wavelength in the media the currents reach a constant magnitude and phase. At one wavelength into the material we can place a boundary thereby reducing model size without any loss in accuracy.

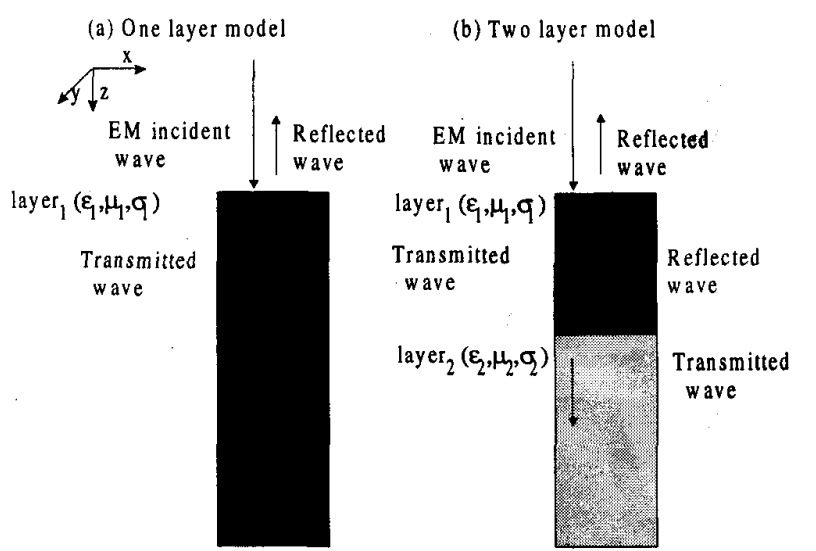

Fig. 5. Propagation path of incident radiation for a single(a) and two layer (b) sub-surface
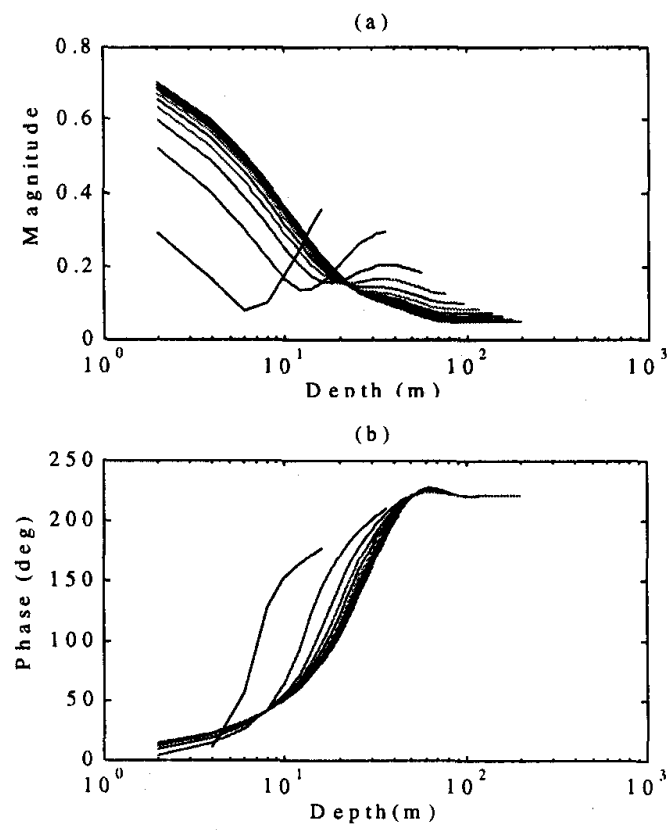

Fig. 6. Eddy currents for rock $\left(\sigma=0.06 \mathrm{~S} / \mathrm{m}, \varepsilon=4.5 \varepsilon_{0}, \delta=13.75, \lambda=\right.$ $86.45 \mathrm{~m}$ ) using models of various depths

\section{Derivation of Surface Impedance}

The electric field component $E_{x}$ is determined from the induced potential that is calculated from the spatial differential of the solved cell currents ie.

$$
E_{x}=\sum_{i=1}^{n} \nabla \cdot V_{i}=\sum_{i=1}^{n} \nabla \cdot \frac{I_{x i}}{Z_{i}}
$$

The magnetic field component is taken from the value at the surface of the region.

$$
H_{y}=H_{i 0}
$$

Hence the surface impedance is calculated from the expression

$$
\mathrm{Z}_{\mathrm{s}}=\frac{E_{x}}{H_{y}}=\frac{\sum_{i=1}^{n} \nabla \cdot \frac{I_{x_{i}}}{Z_{i}}}{H_{i 0}}
$$

\section{NUMERICAL VALIDATION}

\section{A. Steady-state Results}

The source $\mathrm{H}$ field is computed in 1-D using the respective propagation and where appropriate the reflection and transmission coefficients. Fig. 7. compares the theoretical and modelled surface impedance for a number of sub-surface conductivities for a single layer. Fig. 8. shows the theoretical and modelled surface impedance for a 1-D two layered region as a function of ice depth above a rock sub-surface.

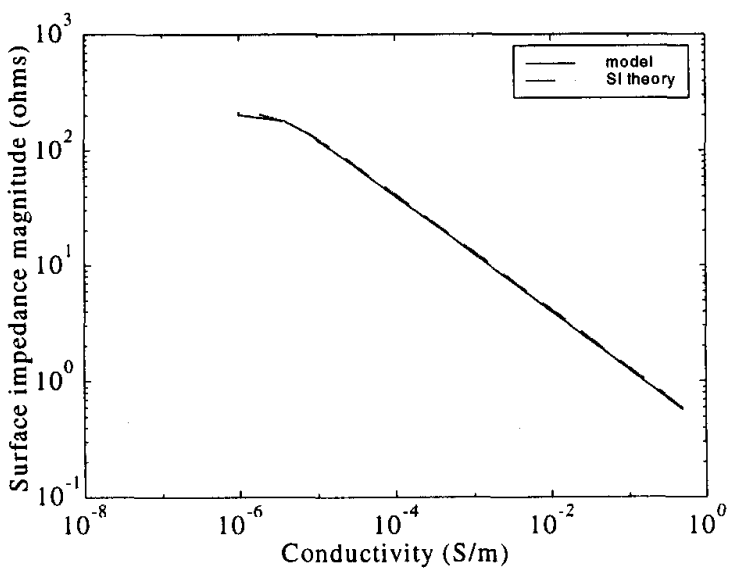

Fig. 7. Surface impedance vs. conductivity for a homogenous earth $\left(\varepsilon=3 \varepsilon_{0}\right.$, $f=22.3 \mathrm{KHz}$ ) using surface impedance theory (1) and the impedance method (11). 
(a)

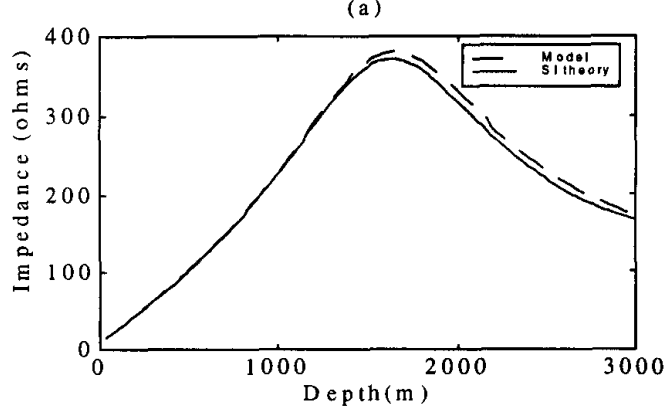

(b)

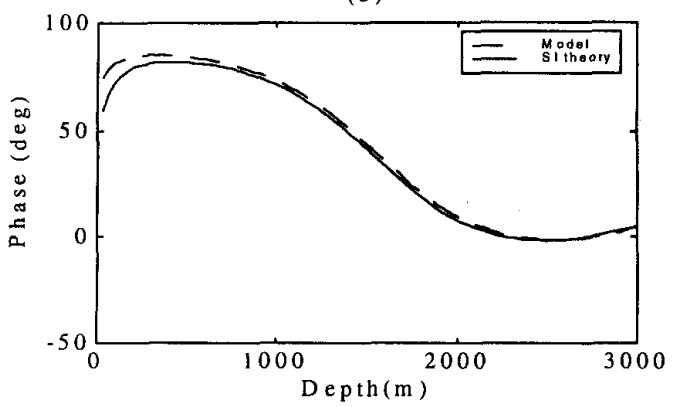

Fig. 8. Surface impedance magnitude (a) and phase (b) as a function of an ice $\left(\sigma=3 \times 10^{-6} \mathrm{~S} / \mathrm{m}, \varepsilon=3 \varepsilon_{0}\right)$ top layer depth above a rock sub-surface ( $\sigma=$ $1.5 \times 10^{-3} \mathrm{~S} / \mathrm{m}, \varepsilon=15 \varepsilon_{0}$ ) for both surface impedance theory (2) and the impedance method (11).

\section{A. Field Validation}

By applying a source magnetic field to an infinite half-space the E field can be computed using the impedance method. The 1-D model is expanded to 2-D to enable the generation of theoretical surface impedance. In particular the behaviour of previously reported surface impedance anomalies near vertical dislocations is modelled and compared with field results [2].

A surface impedance traverse was conducted across a $1 \mathrm{~m}$ $x 1 \mathrm{~m}$ air-gap (formed by summer melt streams) located near the top of a uniform ice sub-surface in the Antarctic. It was simulated using a $32 \times 20$ cell impedance method model. The results are compared in Fig. 9.

\section{CONCLuSIONS}

The impedance method enables the forward modelling of surface impedance profiles that satisfies both surface impedance theory for horizontally layered sub-surfaces and in the vicinity of sub-surface abnormalities.

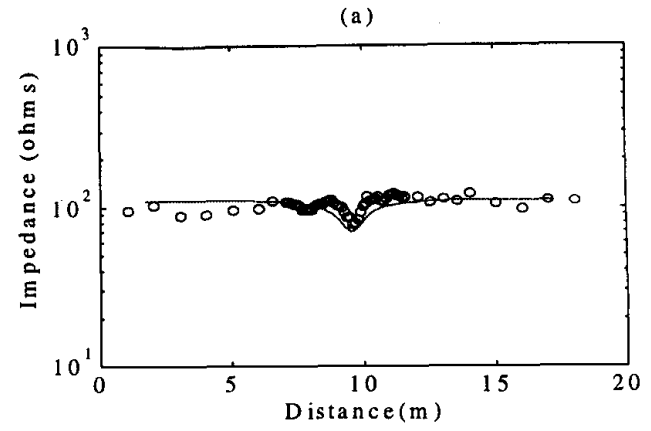

(b)

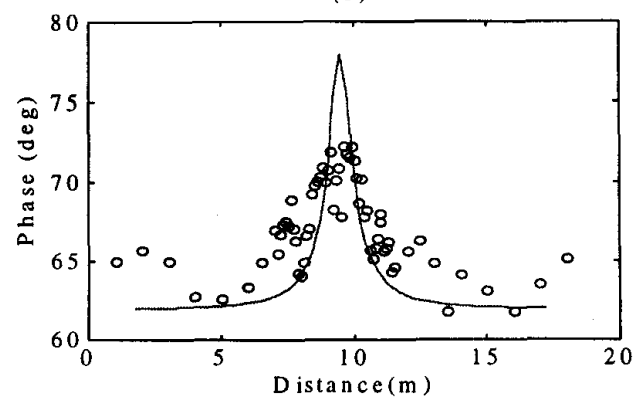

Fig. 9. Surface impedance magnitude (a) and phase (b) across an ice melt stream, measured (o) and model (-) results.

\section{REFERENCES}

[1] D. V. Thiel and F. Neal, "VLF surface-impedance measurements for icedepth mapping in the antarctic," Journal of Glaciology, Vol. 35, No. 120, pp.197-200, 1989.

[2] David. V. Thiel, Daniel James and Peter Johnson, "VLF surface impedance measurements for ice depth mapping - an assessment of some commonly encountered interference effects," Journal of Glaciology, Vol. 42, No. 140 , pp. 33-36, 1992.

[3] David. V. Thiel, "Surface-impedance changes in the vicinity of an abrupt lateral boundary at the earth's surface," IEEE Trans. Geoscience and Remote Sensing, Vol. 28, No. 4, pp. 500-502, July 1990.

[4] W. M. Telford, L.P. Geldart, R.E Sheriff, D.A.Keys, Applied geophysics, Cambridge University Press, Cambridge, 1976.

[5] N. Orcutt and O.P. Gandhi, "A 3-D impedance method to calculate power deposition in biological bodies subjected to time varying magnetic fields," IEEE Trans. Biomed. Eng., Vol. BME-35, pp. 577-583, 1988

[6] W. Xi and M.A. Stuchly, "Induced electric currents in models of man and rodents from $60 \mathrm{~Hz}$ magnetic fields," IEEE Trans. Biomed. Eng., Vol. BME41, No. 11, pp. 1018-1023, 1994.

[7] Daniel A. James and David V. Thiel, "Modelling eddy currents in unbounded structures using the impedance method," Applied Computational Electromagnetics Society, Vol. 12, No. 3, pp. 43-49, November 1997.

[8]J. R. Wait, Electromagnetic waves in stratified media, Second edition, Oxford, Pergamon Press 1991. 